\title{
Optimization of dyeing condition and its dyeing on Palmyrah (Borassus flabellifer) leaves
}

\author{
Subajini Mahilrajan ${ }^{1} *$, Jeyarani Nandakumar ${ }^{2}$, Robika Kailayalingam ${ }^{1}$ and \\ SriThayalan SriVijeindran ${ }^{1}$ \\ ${ }^{1}$ (Palmyrah Research Institute, Kaithady, Jaffna, Sri Lanka \\ ${ }^{2}$ (Department of Botany, University of Jaffna, Sri Lanka)
}

\begin{abstract}
Palmyrah leaf based articles have been dyed mostly with direct dyes till today, which always have problems to dyers is that complications in dye reproducibility. Therefore this study was concluded about standardize the dyeing variables of alkaline dye. The optimum wave length was $600 \mathrm{~nm}$, out of a set of wave length ranging from 400-700 $\mathrm{nm}$ on the basis of highest absorbance. Five dye concentrations (0.1-0.5g/l) were tried and $0.3 \mathrm{~g} / \mathrm{l}$ dye concentrations were selected on the basis of dye absorption. Likewise for the good dye absorption optimum temperature, optimum time and optimum leaf: liquor ratio was $100^{\circ} \mathrm{C}, 30$ min and 1:20 respectively. Significantly higher dye absorption and wash fastness was observed leaves with beaching than without bleaching while there was no significant different among the property of light fastness. Effect of auxiliary was studied with sodium chloride, sodium carbonate and naphthalene and naphthalene was selected as the best auxiliary based on the light fatness properties.
\end{abstract}

Keywords: Bleaching, Dye absorption, Fastness, Naphthalene, and Palmyrah leaf

\section{INTRODUCTION}

Dyeing is a complex process, where numbers of variables are involved. Dyeing process is broadly governed by fabric, dye and time, temperature, $\mathrm{pH}$ of the fabric and liquor, type of auxiliary used etc. Any minor variation in any of these variables causes problems in dye reproducibility, though it is possible to achieve reproducibility in dyeing results. By standardizing each and every variable consistent reproducible results can be achieved.

The temperature of the dye bath affects the affinity of the dye molecules towards fibre, rate of hydrolysis, migration and covalent bond formation, therefore the dyeing temperature selected must be as per the dye type. Percentage of dye absorption increased with increase in temperature and after that, the dye absorption decreased [1], [2]. Auxiliaries play an important role in dyeing of reactive dyes. They help in better exhaustion of the dyes. References [3], [4] reported that the addition of electrolyte to the dye liquor of anionic dye increased the uptake of dye by the fabric. The electrolyte used in dyeing dissociates completely in aqueous dye liquor. For entering into the fabric, the charge on surface (negative in fabric) will have to be neutralized since both anionic dyes and fabric have the same charge. Sodium ion $(\mathrm{Na}+)$ from sodium chloride is cationic and in the dye liquor is attracted by the negatively charged fabric. By bonding the sodium cations neutralize the anionic surface charge of the fabric. Now the neutralized fabric can attract the organic dye molecules which have a greater affinity for the fabric than the aqueous solution. Dyes molecules permanently bind with cellulose based fibers (cotton, rayon, hemp, linen) as well as leaves, when the $\mathrm{pH}$ is raised. Soda ash (sodium carbonate) is generally used to raise the $\mathrm{pH}$ and is either added directly to the dye or in a solution of water in which garments are soaked before dyeing [5]. Because of this reason alkaline dyes were used for this study.

Some of the problems are identified in handicrafts design centers. Leaf based articles have been dyed mostly with direct dyes till today, which always have problems to dyers is that complications in dye reproducibility as well as consumers as these articles fades very easily so an attempt has been made to standardize the dyeing process for leaves using alkaline dye which have good colour fastness.

Objective of the study is dyeing process to reduce the fade of dyed Palmyrah Leaves with the aim of optimization of alkaline dye and its dyeing on Palmyrah leaves

\section{DESCRIPTION OF RESEARCH}

Standardization of dyeing process for palmyrah tender leaves with alkaline dye: Experiments were carried out to optimize dye concentration, dyeing time, dyeing temperature and leaf liquor ratio for dyeing of palmyrah leaf with alkaline dye. 


\subsection{Determination of optimum wave length}

For determining the optimum wavelength $1 \mathrm{ml}$ of dye was diluted to 200 times and absorbance of the solution was taken on a spectrophotometer at different wave lengths from 400 to $700 \mathrm{~nm}$. The wavelength reflecting the highest optical density was selected.

\subsection{Optimization of dye concentration}

For determining the optimum dye concentration, five different concentrations of reactive dye i.e. 0.1, $0.3,0.5,0.75$ and $1 \mathrm{~g} / \mathrm{L}$ were taken and samples were dyed at $95-100^{\circ} \mathrm{C}$ for 30 minutes. Absorbance of dye solutions before and after dyeing was recorded at optimum wavelength. The dye solution giving the maximum dye absorption was taken as optimum dye concentration.

\subsection{Optimization of dyeing temperature}

To optimize dyeing temperature, dyeing was carried out using optimum concentration of dye at five different temperatures i.e. $70,80,90$ and $100^{\circ} \mathrm{C}$. The temperature giving maximum dye absorption was taken as the optimum dyeing temperature.

\subsection{Optimization of dyeing time}

The leaves samples were dyed using optimum dye concentration for five different time durations i.e. 10, 20, 30 and 40 minutes. The optimum dyeing time was selected on the basis of maximum dye absorption.

\subsection{Optimization of leaf liquor ratio}

To ascertain the optimum dyeing liquor leaf (L: R) ratio, five samples were dyed at the optimum concentration, temperature and time at 1:10, 1:15, 1:20, 1:25 and 1:30. Optimum dyeing leaf liquor (L: R) ratio was decided on the basis of maximum dye absorption.

\subsection{Effect of bleaching agent}

This palmyrah leaf contained high amount of lignin therefore the leaves samples were heated at $100^{\circ} \mathrm{C}$ for $10 \mathrm{~min}$ with hydrogen peroxide $(4 \mathrm{ml} / \mathrm{l})$ and then dyed using optimum dyeing condition. For control treatment was done without pre heating and hydrogen peroxide. The optimum dyeing was selected on the basis of maximum dye absorption.

\subsection{Effect of auxiliaries}

Different auxiliaries such as sodium chloride, sodium carbonate and naphthalene $(10 \mathrm{~g} / \mathrm{l})$ were added in dye bath separately. Best auxiliary was decided on the basis of maximum dye absorption.

\subsection{Fastness testing}

Wash fastness of the leaves dyed without bleaching, dyed with bleaching, dyed with naphthalene, dyed with $\mathrm{NaCl}$, dyed with $\mathrm{Na}_{2} \mathrm{CO}_{3}$ and dyed with bleaching and Naphthalene under optimized condition was tested according to ISO $105-\mathrm{CO} 3$ method. The above dyed leaves were washed in soap solution (Na salted) for 30 min at room temperature. Half of the dyed leaf was draped with black paper and covered with glass slide then placed on direct sun light for $24 \mathrm{~h}$. Then light fastness was tested by colour scale is for assessing changes in colour of leaf in colour fastness tests, for example the leaf consists of five number of colour each representing a visual difference and contrast. The fastness rating goes step-wise from:

Note $5=$ no visual change (best rating)

Note $1=$ a large visual change (worst rating).

The colour scale has the 5 possible values: 5, 4, 3, 2 and 1

\section{Results And Discussion}

Basic or cationic dyes on ionization give coloured cations and form an electrovalent bond with the $\mathrm{COOH}$ group of wool and silk. These dyes are applied from neutral to mildly acidic $\mathrm{pH}$. These dyes have poor light fastness.

\subsection{Determination of wave length:}

Optimum wave length is the wave length at which maximum absorbance was observed. The absorbance was recorded from 400 to $700 \mathrm{~nm}$. The maximum absorbance was observed at $600 \mathrm{~nm}$ hence this wave length was selected for further studies (Fig: 1). 


\subsection{Optimization of dye concentration}

On the basis of dye absorption to optimize the dye concentration $0.1,0.2,0.3,0.4$ and $0.5 \mathrm{~g} / \mathrm{L}$ dye concentration were taken (Fig: 2$)$. There was no significant difference $(\mathrm{P}>0.05)$ between treatments with higher absorbance of dye with the concentration of $0.3,0.4$ and $0.5 \mathrm{~g} / \mathrm{L}$. Hence $0.3 \mathrm{~g} / \mathrm{L}$ dye concentration was selected because mean difference between 0.5 and $0.3 \mathrm{~g} / \mathrm{L}$ is greater than mean difference between 0.3 and $0.4 \mathrm{~g} / \mathrm{L}$ and also $0.3 \mathrm{~g} / \mathrm{L}$ is cost effective concentration when compared with higher absorbance concentrations. It is found that dye absorption by cotton fabric increased with the increase in dye concentration in the dye bath because the absolute quantity of the absorbed dye increases while the relative quantity diminishes [6].

\subsection{Optimization of dyeing temperature}

Dyeing temperature is the temperature that is suitable for dye absorption and fixation of dye on the leaf material. For optimizing dyeing temperature, dyeing was carried out at four different temperatures i.e. 70, 80, 90 and $100^{\circ} \mathrm{C}$. The percent dye absorption at different temperatures is given in TABLE: 1 . Mean absorption value for temperature 90 and $100^{\circ} \mathrm{C}$ is significantly higher than that of temperature 70 and $80^{\circ} \mathrm{C}$, while there was no significant difference between the absorptions of temperature for 90 and $100^{\circ} \mathrm{C}$. Therefore $100^{\circ} \mathrm{C}$ (boiling) was selected as the optimum dyeing temperature. Ali [7] reported that this increase in dye uptake can be attributed to better dye exhaustion at higher temperature.

\subsection{Optimization of dyeing time}

Dyeing time is the time required to get the dye fixed on palmyrah leaves. The effect of dyeing time on absorbance is shown in TABLE 2. Mean absorption value for time at 30 and 40min is significantly higher than that of time at 10 and $20 \mathrm{~min}$, while there was no significant difference between the absorptions of time for 30 and 40min. Therefore 30min was selected as the optimum dyeing time for better exhaustion. Longer the dyeing time create higher absorbance until dye exhaustion attains equilibrium [7].

\subsection{Optimization of Leaf liquor Ratio}

The effect of leaf liquor ratio on absorbance is shown in figure 2. Mean absorption value for $\mathrm{L}: \mathrm{R}$ for $1: 10,1: 15$ and $1: 20$ is significantly higher than that of $\mathrm{L}: \mathrm{R}$ for $1: 25$ and 1:30, while there was no significant difference between the absorptions of $\mathrm{L}: \mathrm{R}$ for for 1:10, 1:15 and 1:20 (Fig: 3). Therefore 1:20 was selected as the optimum dyeing L: R for better exhaustion. Higher absorbance value at lower L: R may be explained by crowding of dye molecule at lower L: $\mathrm{R}$ resulting in increased dye exhaustion of the leaf [7].

\subsection{Effect of bleaching agent}

The leaves samples were heated at $100^{\circ} \mathrm{C}$ for $10 \mathrm{~min}$ with hydrogen peroxide $(4 \mathrm{ml} / \mathrm{l})$ and then dyed using optimum dyeing condition. For control treatment dyeing was done without preheating (Fig: 4). Mean percentage of dye absorption was significantly higher for dyed with bleaching than dyed with non-bleaching method.

\subsection{Effect of auxiliaries}

A mordant is more important than the dye itself. Moreover, the ideal mordant for bulk use should produce appreciable colour yield in practicable dyeing conditions at low cost, without seriously affecting physical properties of fibre or fastness properties of the dyes. Different auxiliaries such as sodium chloride, sodium carbonate and naphthalene $(10 \mathrm{~g} / \mathrm{l})$ were added in dye bath separately.

Percentage of dye absorption with $\mathrm{Na}_{2} \mathrm{CO}_{3}$ was significantly higher than other auxiliary this may be due to de-colouration of dye on alkaline medium. Among the $\mathrm{NaCl}$ and naphthalene there was no significant different between on dye absorption while de-colouration to direct sunlight for $24 \mathrm{~h}$ was higher for $\mathrm{NaCl}$ than naphthalene (Fig: 5). Therefore Naphthalene was selected for further study.

\subsection{Fastness testing}

Wash fastness for colour scale for dyed with bleaching and dyed with bleaching and Naphthalene was showed higher than other treatments (TABLE 3) while those treatments showed less light fastness colour scale although dyed with naphthalene showed good light fastness (Fig: 6) property. 


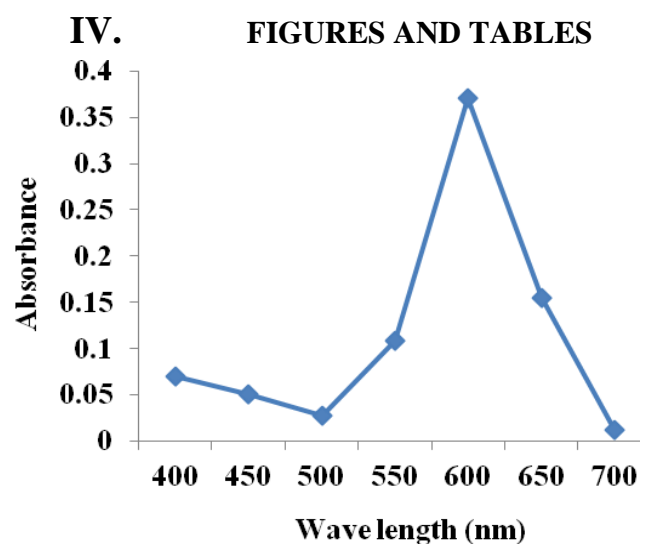

Fig 1: Wave length of alkaline dye (Malachite green).

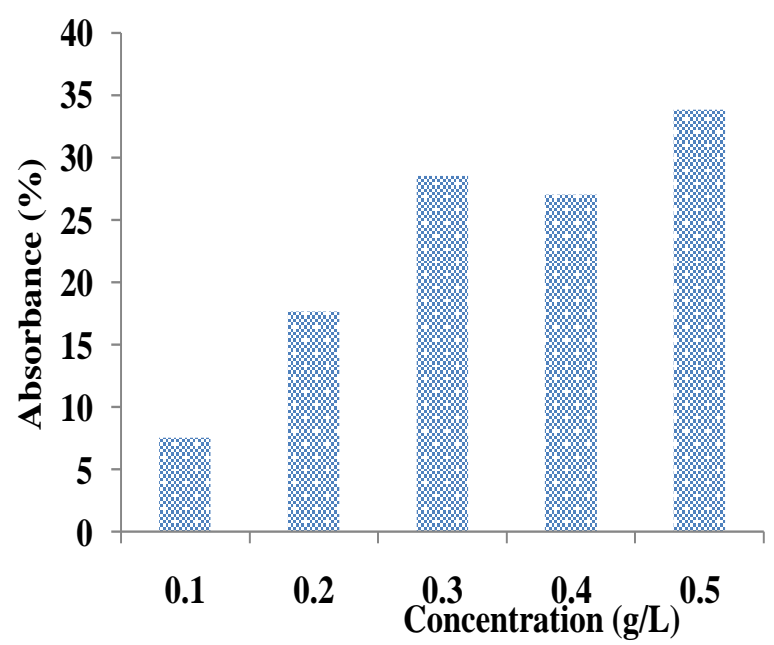

Fig 2: Optimization of dye concentration on the basis of dye absorption

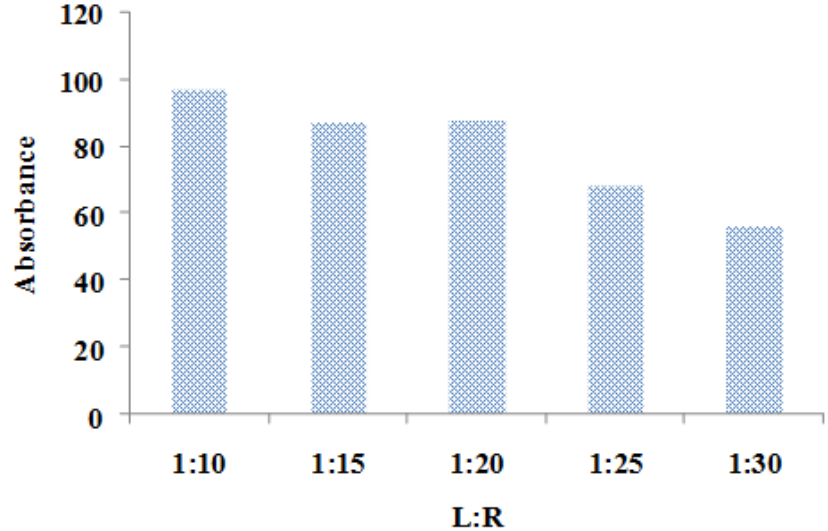

Fig 3: Optimization of leaf liquor ratio on the basis of dye absorption 


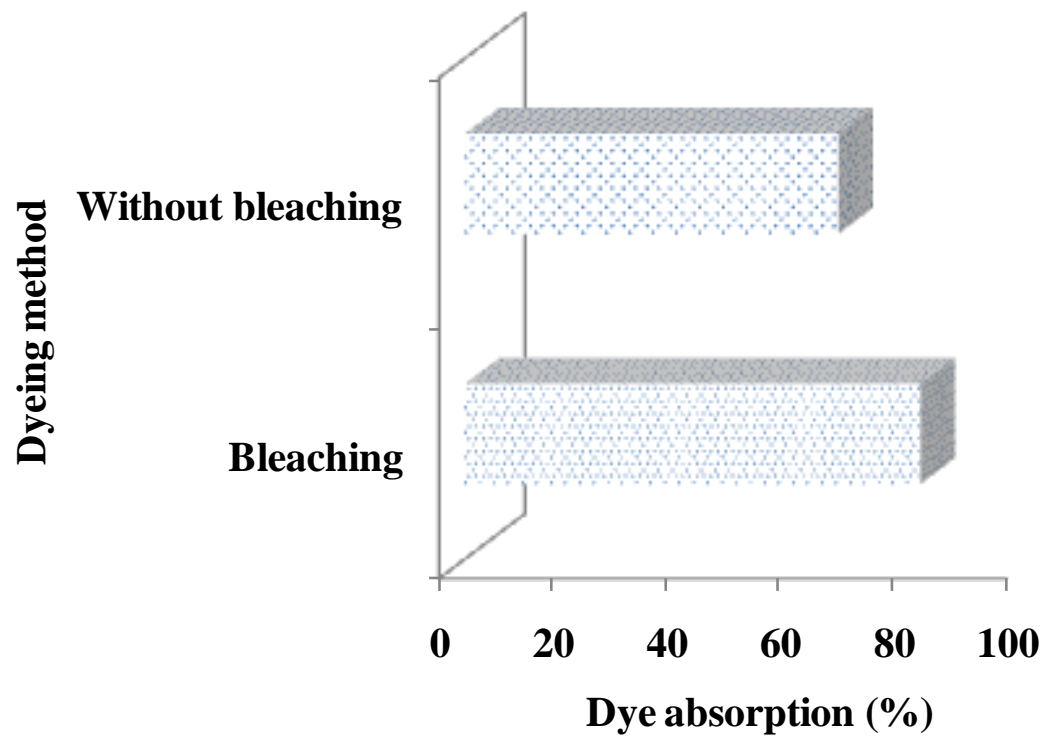

Fig 4: Effect of bleaching on dye absorption

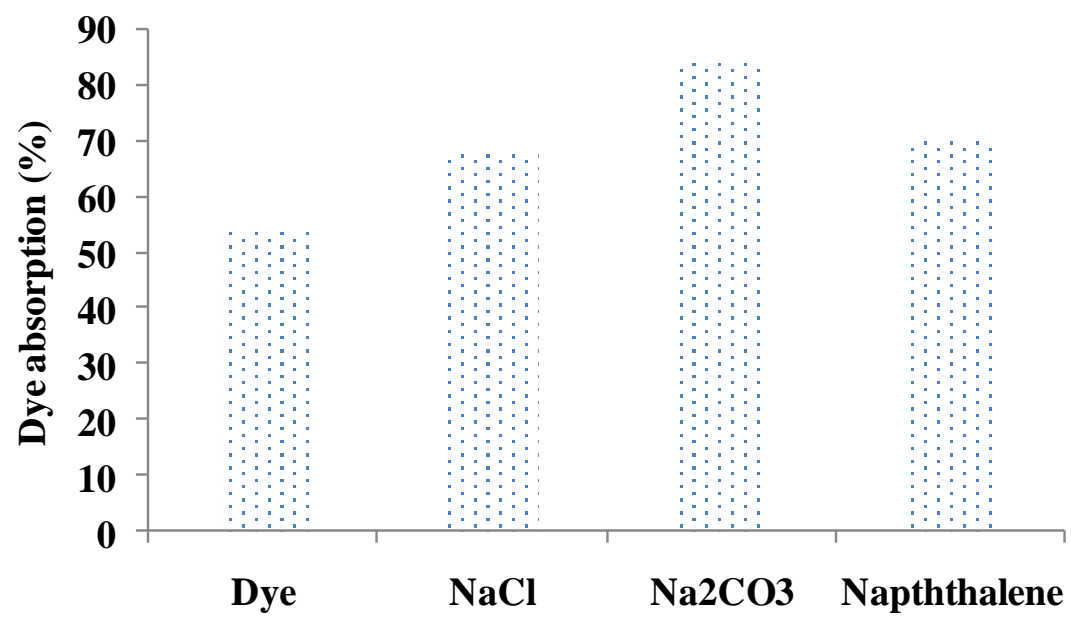

\section{Type of Auxiliary}

Fig 5: Effect of auxiliary on dye absorption

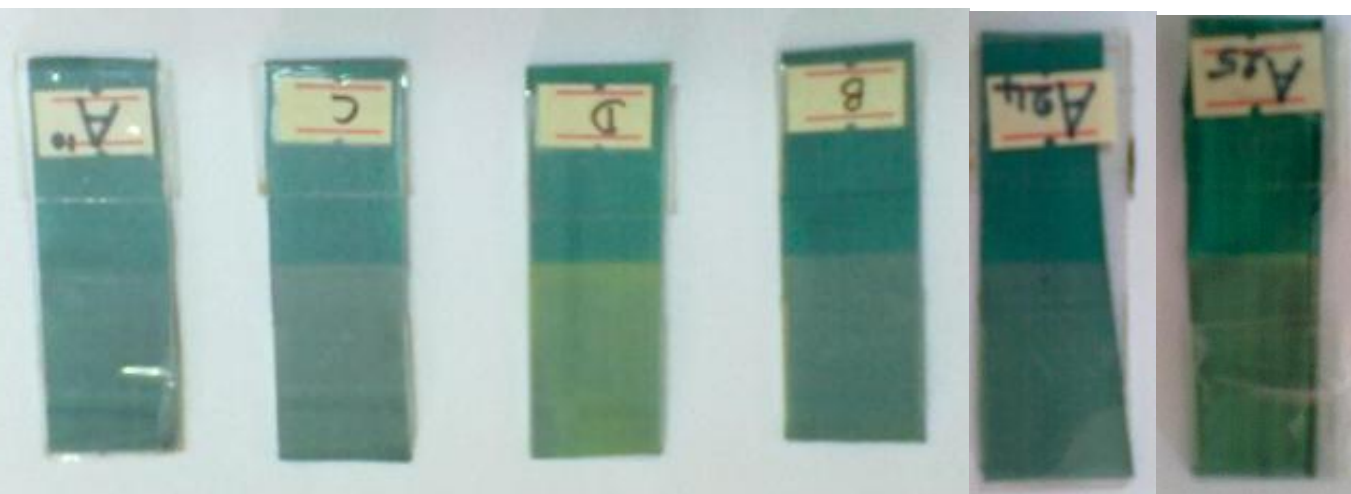

Fig 6: Dyed without bleaching $(B)$, dyed with bleaching $\left(A_{24}\right)$, dyed with naphthalene $\left(A_{10}\right)$, dyed with $\mathrm{NaCl}(\mathrm{C})$, dyed with $\mathrm{Na}_{2} \mathrm{CO}_{3}(\mathrm{D})$ and dyed with bleaching and Naphthalene $\left(\mathrm{A}_{25}\right)$. 
TABLE 1: Optimization of dyeing temperature on the basis of dye absorption

\begin{tabular}{cc}
\hline Temperature $\left({ }^{\circ} \mathbf{C}\right)$ & Absorbance $(\%)$ \\
\hline 70 & 17 \\
80 & 21 \\
90 & 64 \\
100 & 86 \\
\hline
\end{tabular}

TABLE 2: Optimization of dyeing temperature on the basis of dye absorption

\begin{tabular}{cc}
\hline $\begin{array}{c}\text { Time } \\
(\mathbf{m i n})\end{array}$ & Absorbance (\%) \\
\hline 10 & 70 \\
20 & 75 \\
30 & 86 \\
40 & 91 \\
\hline
\end{tabular}

TABLE 3: Colour score for fastness test of different dyed method

\begin{tabular}{ccc}
\hline Method of dyeing & $\begin{array}{c}\text { Wash } \\
\text { fastness }\end{array}$ & $\begin{array}{c}\text { Light } \\
\text { fastness }\end{array}$ \\
\hline Dyed without bleaching & 4 & 3 \\
Dyed with bleaching & 5 & 3 \\
Dyed with naphthalene & 4 & 4 \\
Dyed with $\mathrm{NaCl}$ & 3 & 3 \\
Dyed with $\mathrm{Na}_{2} \mathrm{CO}_{3}$ & 2 & 2 \\
Dyed with bleaching and Naphthalene & 5 & 3 \\
\hline
\end{tabular}

\section{CONCLUSION}

This study could be conclude for optimum dyeing with good dye absorption accordingly leaf materials have to dyed with the concentration of $0.3 \mathrm{~g} / \mathrm{l}$, Temperature $100^{\circ} \mathrm{C}$, Time of dyeing 30 min and leaf: Liquor ratio 1: 20. Besides dyed with bleaching was showed best wash fastness and dyed with naphthalene showed good light fastness.

\section{Acknowledgements}

The authors thank Ministry of Traditional Industries and Small Enterprise Development, Sri Lanka for the financial support.

\section{REFERENCES}

[1] J. Taylor, The dyeing of cotton with hetero bi-functional reactive dyes containing both a monochlorotriazinyl and a chloroacetlyamins reactive groups, Dyes and Pigments. 51(6), 2001, 145-152.

[2] S. Bae, H. Motomura, and Z. Morita,.Adsorption behavior of reactive dyes on cellulose. Dyes and Pigments. 40(1),1998, 37-55.

[3] S. Alam , G.M. Khan, and S.M. Razzaque, Dyeing of cotton fabrics with reactive dyes and their physico-chemical properties, Indian Journal of Fibre and Textile Research, 33(1), 2008, 58-65.

[4] A. Farha, S.A. Gamal, A.M. and Shallam, H.B, Sodium edate and sodium citrate as an exhausting and fixing agents for dyeing cotton fabric with reactive dyes and reuse of dyeing effulent, Journal of American Science. 6(10), 2010, 109-112.

[5] Anonymous. 2007. Reactive dye. http://www.en.wikipedia.org/wiki/ Reactive_dye. Retrieved on 16.6.2011.

[6] J. Singla, S. S. J.Singh, and N. M. Rose, Standardization of Dyeing Variables of Reactive Dye for Tie and Dye on Cotton, Home Science, 1: 5, 2012. $2277-8160$.

[7] S. Ali, T. Hussain and R. Nawaz, Optimization of alkaline extraction of natural dye from Henna leaves and its dyeing on cotton by exhaust method, Journal of Cleaner Production, 2008, 1-6. 